\title{
A Novel Sparse Data Reconstruction Algorithm for Dynamically Detect and Adjust Signal Sparsity
}

\author{
Dongxue Lu ${ }^{1}$,Zengke Wang* \\ ${ }^{1}$ Electronic Payment Resarch Institute, China Unionpay Co.Ltd, Shanghai,201210,China \\ E-mail:2981841943@qq.com \\ *College of Electronic Information and Optical Engineering,Nankai University,Tianjin,300350,China \\ E-mail:wangzk@mail.nankai.edu.cn
}

Received: December 28, 2020. Revised: May 23, 2021. Accepted: June 14, 2021. Published: June 25, 2021.

\begin{abstract}
This paper proposed a novel algorithm which is called the joint step-size matching pursuit algorithm (JSTMP) to solve the issue of calculating the unknown signal sparsity. The proposed algorithm falls into the general category of greedy algorithms. In the process of iteration, this method can adjust the step size and correct the indices of the estimated support that were erroneously selected in a dynamical way. And it uses the dynamical step sizes to increase the estimated sparsity level when the energy of the residual is less than half of that of the measurement vectory. The main innovations include two aspects: 1) The high probability of exact reconstruction, comparable to other classical greedy algorithms reconstruct arbitrary spare signal. 2) The $\sinh ()$ function is used to adjust the right step with the value of the objective function in the late iteration. Finally, by following this approach, the simulation results show that the proposed algorithm outperforms stateof-the-art similar algorithms used for solving the same problem.
\end{abstract}

Keywords - compressed sensing; sparse data; dynamic step size; function model; data sparsity.

\section{INTRODUCTION}

$\mathrm{n}$ recent years, the research of compressed sensing(CS) [1-3] has received more attention as a mean to process the sparse signal (i.e., the number of nonzero elements in the vector is small). CS is a newly developed signal processing technique for efficiently acquiring and reconstructing a signal by finding solutions to underdetermined linear systems. Sparse signal reconstruction technology has important applications and extensive research in computer science and electronic science. E.g. Single pixel camera developed by MIT, magnetic resonance imaging, and wireless sensor network technology. Zhu [4] take the structured perturbation into account and propose the perturbation considered vector approximate message passing (PC-VAMP) algorithm. Qiu [5] proposed a joint lowrank and sparsity priors' constrained model for ISAR imagingwith various sparse data patterns. And it also has important applications in deep learning. E.g. Leong [6] present group lasso as an efficient method for obtaining robust cluster expansions (CEs) of multicomponent systems. Li [7] proposed a deep learning method to accurately reconstruct images for previously solved and unsolved CT reconstruction problems with high quantitative accuracy. Particularly, accurate reconstructions were achieved for the case when the sparse view reconstruction problem.

CS theory can exactly recover the sparse signal or compressible signals by a sampling rate which does not satisfy the Nyquist-Shannon sampling theorem. But many papers have demonstrated that CS can effectively get the key information from sample value with a small number of non-correlative measurements. The main idea of CS is to make compression and sampling run at the same time. CS technique can reduce the hardware requirements, further reduce the sampling rate, improve the signal quality, save on signal processing and transmission costs. Nowadays, CS has been widely used in wireless sensor networks, information theory, image processing, earth science, optical/microwave imaging, pattern recognition, wireless communications, atmosphere, geology and other fields.

CS theory mainly includes three aspects: 1) The sparse representation of signals; 2) The design of the measurement matrix; 3) Signal reconstruction algorithm. One of the most important aspects is the design of the reconstruction algorithm. It is the huge challenge for the researchers to propose a quick reconstruction algorithm with reliable accuracy. At present, the studies of domestic and foreign scholars have proposed many reconstruction algorithms. There are two important kind of the reconstruction algorithm. One is greedy algorithm, the other is convex optimization algorithm. A series of iterative greedy algorithms received significant attention due to their low complexity and simple geometric interpretation. Briefly, the greedy algorithm selects the support (the set of nonzero elements) of the sparse signal vector $\boldsymbol{x}$ in an iterative manner. The algorithms in this category include matching pursuit(MP), orthogonal matching pursuit(OMP), Stagewise OMP(StOMP) [8], Regularized OMP(ROMP), Subspace Pursuit(SP) [9], Compressive sampling OMP(CoSaMP) and so on. The basic idea of those method is to find the support of the sparse signal vector $\boldsymbol{x}$. In each iteration, one or many indexs of the column are selected for testing based on the correlation values between the sensing matrix $\boldsymbol{\Phi}$ and the new residual. If those indexs are reliable, they will be added to the final support set to recover the original signal. The pursuit algorithms iterate this procedure until all indexs in the correct support set are included in the final support. The convex optimization algorithm solves a much easier $\boldsymbol{l}_{\boldsymbol{l}}$-norm minimization problem based on linear programming (LP), such as basis pursuit(BP) which requires high computational complexity to achieve exact reconstruction.

In this paper, we propose a novel algorithm to reconstruct the sparse 
signal which is called the joint step-size matching pursuit Penrosepseudoinverse [16] of $\Phi$. And the $\Phi^{\prime}$ represents the matrix algorithm(JsTMP). The proposed algorithm can adjust the step size and transposition. The projection of the measurement $\boldsymbol{y}$ onto span $\left(\Phi_{S}\right)$ is correct the indices of the estimated support that were erroneously defined as selected in a dynamical way. The simulation results show that the proposed algorithm outperforms state-of-the-art similar algorithms used for solving the same problem.

The rest of this paper is organized as follows: Section 2 introduces the compressive sensing model and the Preliminaries of JsTMP algorithm. In Section 3, we discuss the performance of proposed algorithm and conclude the paper in Section 4.

\section{RELATED WORKS}

\section{A. Compressive Sensing Model}

Compressive sensing acquires sparse signals at a rate significantly below Nyquist rate. Signal is sparse or sparse is the application of compressed sensing prior information. Prior information using CS technology is that signal is sparse or compressible. $K$-sparse signal vector $\boldsymbol{x}$, i.e, n-dimensional vector having at most $K$ nonzero elements. It is the basic principle that the sparse signals $x \in R^{n}$ can be reconstructed from the measurement $\boldsymbol{y}$. The measurement is expressed as

$$
y=\Phi x
$$

$\Phi$ is the sensing matrix $\left(\Phi \in R^{m \times n}, m \ll n\right)$. Since the $m \ll n$ for the model, the system in (1) can be regard as the underdetermined system or sick system. Obviously, it is impossible to recover the original sparse signal from $\boldsymbol{y}$ by using the conventional "matrix inverse" transform of the sensing matrix $\Phi$. But we can make full use of the prior information on the signal sparsity and a condition imposed on $\Phi$ then $\boldsymbol{x}$ can be correctly reconstructed by solving the $\boldsymbol{l}_{\boldsymbol{l}}$-norm problem as follows:

$$
\min \|x\|_{1} \quad y=\Phi x
$$

The sensing matrix $\Phi$ must satisfy the restricted isometry property(RIP) [10-12]. It ensures that the sensing matrix will not map two different $K$ sparse signals into the same set. Sensing matrix falls broadly into three categories: random measurement matrix, partial random measurement matrix and deterministic measurement. They all satisfy the restricted isometric principle. The commonly used sensing matrixes include the Gaussian random matrix [13], Bernoulli random matrix, Fourier random matrix, Toeplitz matrix [14-15], etc. $K$ is the signal sparsity. The reconstruction accuracy of the $\boldsymbol{l}_{\boldsymbol{l}}$-norm is limited in terms of the RIP formally defined below.

Definition 1(RIP): A matrix $\Phi \in R^{m \times n}$ is described to satisfy the restricted isometry property(RIP) with the parameters $\left(K, \delta_{S}\right)$.For $K<$ $m, 0 \leq \delta_{S}<1$ and $S \subseteq\{1,2,3, \ldots, \mathrm{n}\}$, the matrix $\Phi_{S}$ consists of the columns of $\Phi$ with indices $\mathrm{S} \in S$. Such that $\|S\|<K$ and $x \in R^{|S|}$ ,one has

$$
\left(1-\delta_{S}\right)\|x\|_{2}^{2} \leq\left\|\Phi_{S} x\right\|_{2}^{2} \leq\left(1+\delta_{S}\right) \|\left(1+\delta_{S} \|_{2}^{2}\right.
$$

Generally, if the $\delta$ is very close to one, then it is possible that $\left\|\Phi_{S} x\right\|_{2}^{2} \approx 0$.so the measurement $y$ can not preserve any information on $\boldsymbol{x}$ (i.e, $\boldsymbol{x}$ is nullspace of the sensing matrix $\Phi$ ). As a result, it is nearly impossible to reconstruct the sparse signal $\boldsymbol{x}$ by using any greedy algorithms when $\delta_{S} \approx 1$.

\section{B. Sparse signal approximation method}

In order to describe the main steps of the JsTMP algorithm, we introduce the notation of the projection of a vector and its residue.

Definition 2(Projection and Residue): Let $y \in R^{m}, \Phi_{S} \in R^{m \times|S|}$. If $\Phi$ is full column rank, then $\left(\Phi^{\prime} \Phi\right)^{-1} \Phi^{S}$ is the Moore-

$$
y_{p}=\Phi_{s} \Phi_{S}^{\dagger} y
$$

where

$$
\Phi_{S}^{\dagger}=\left(\Phi_{S}^{\prime} \Phi_{S}\right)^{-1} \Phi_{S}^{\prime}
$$

It can be seen from the equation (4) that the reconstruction result of the sparse signal $\boldsymbol{x}$ can be expressed as

$$
\hat{x}=\Phi_{S}^{\dagger} y=\left(\Phi_{S}^{\prime} \Phi_{S}\right)^{-1} \Phi_{S}^{\prime} y
$$

The residue of the projection vector $\boldsymbol{y}$ equals

$$
y_{r}=\left(I-\Phi_{\mathrm{S}} \Phi_{\mathrm{S}}^{\dagger}\right) y
$$

Suppose that $\Phi_{S}^{\prime} \Phi_{S}$ is invertible. The projection of $y$ onto $\operatorname{span}\left(\Phi_{\mathrm{S}}\right)$ is defined as

$$
y_{p}=\Phi_{\mathrm{S}} \Phi_{\mathrm{S}}^{\dagger} y
$$

After each iteration, the $\boldsymbol{I}$ stands for the unit matrix, in order to illustrate the relationship between projection and residue of the vector $y$ before and after iteration, we draw a diagram below. See the Fig.1 for the definition.

As can be seen from Fig.1, the reconstruction result of the sparse signal obtained by using equation (6) can ensure that the residue is perpendicular to the hyperplane. This means that the residue of each iteration are minimized. And that can speed up the convergence of the algorithm.

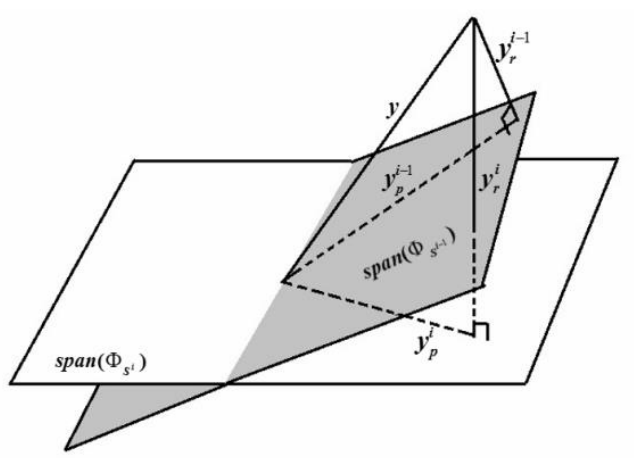

Fig. 1 In each iteration, a T-dimensional hyper-plane closer to $y$ is obtained.

\section{C.The Preliminaries of JsTMP Algorithm}

In this section, we will provide a summary of the proposed algorithm.In particular, If the signal sparsity and length are set to $K$ and $n$. In sparse signal reconstruction, sparsity level is an important piece of information that determines whether the original signal can be exactly reconstructed. However, in practical applications, we can not know the sparsity level in advance. If we know the signal sparsity, we can determine how many indices the recover algorithm needs to choose to reconstruct the original signal. This paper proposed JsTMP algorithm takes full advantage of the fixed steps and dynamic steps, and creatively uses $\sinh ()$ function model to get the sparsity level accurately. In the early stage, the sparsity of the signal can be close quickly by using a larger fixed step. In the latter stage, the $\sinh ()$ function model is used to dynamically adjust the step size to get the exact sparsity level. 
We supposed that the size of the support set at the i-th stage is equal to $K i$. The residual $r_{i}=y-\Phi_{\mathrm{Ki}} \Phi_{\mathrm{Ki}}^{\dagger} y$ decreases gradually with the increase of the Ki.Let $\alpha=\operatorname{norm}\left(r_{i}\right) / \operatorname{norm}(y)(0 \leq \alpha \leq 1)$, and a threshold $\beta=1-\alpha(0 \leq \beta \leq 1)$. The norm $\left(r_{i}\right)$ stands for residual energy of the $\mathrm{i}$-th iteration. In the later stages of signal reconstruction, the step sizes is dynamically adjusted with the gradual decrease of $\alpha$. When $\beta \leq 0.55$, the JsTMP algorithm uses a fixed-step strategy to speed up convergence. When $\beta>0.55,\left\lceil L_{i}\right\rceil$ can be used to dynamically adjust the step sizes.

$$
L_{i}=\left(-\sinh \left(5^{*} \beta-5\right) / 10\right)+1
$$

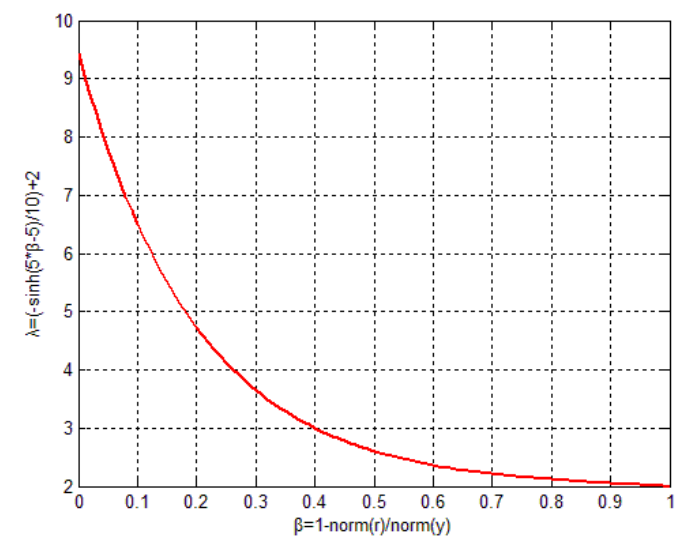

Fig. 2 Step-size changing ratio $\lambda$ versus residual to measurement energy ratio $\beta$

A diagram of the proposed algorithm is depicted in Fig. 3. The initial fixed step size $L_{0}$ can set to any constant. But this constant had better not to be greater than 10. As show in Fig. 3, in the iteration, $r_{i}$ represents the residuals of the i-th iteration, $\rho$ is the end threshold. The JsTMP algorithm uses the fixed step size and dynamic step size to adjust the number of selected index, while using backtracking strategy to remove the wrong index. An index, which is considered reliable in some iteration but shown to be wrong at a later iteration, can be added to or removed from the estimated support set at any stage of the recovery process.

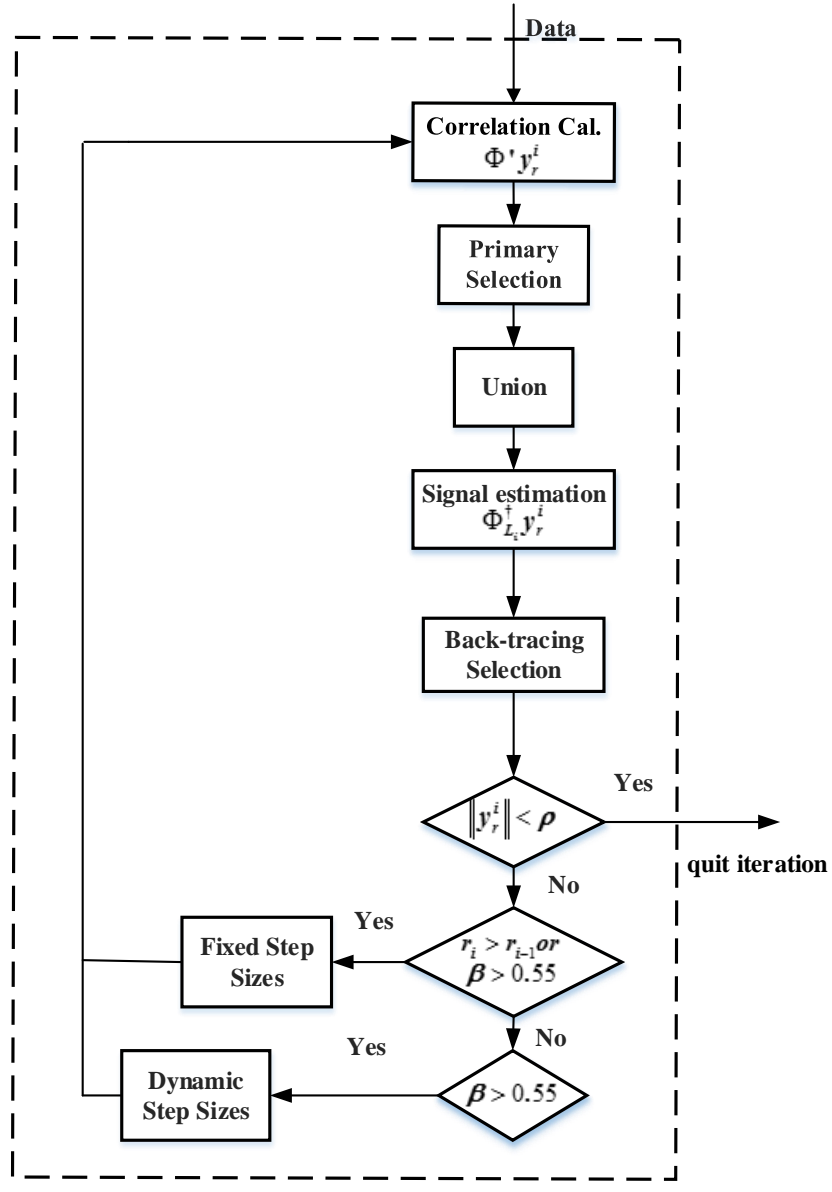

Fig.3 Description of the proposed reconstruction algorithms for $K$ sparsesignals.

\section{THE PROPOSED JSTMP ALGORITHM}

The main steps of the JsTMP algorithm are summarized in Table 1 .

Table 1. The proposed JsTMP Algorithm

Input: Sensing matrix $\Phi$, measurement vector $y$, Initialization step size $L_{0}$

Output: A $K$-sparse approximation $\hat{x}$ of the original vector $x$

I.Initialization:
1. $\hat{x}_{0}=0$
(Initial approximation)
2. $r_{0}=y$
(Initial residue)
3. $L=L_{0}$
(Initial step size)

II. Repeat:

1. $i=i+1$

(The number of iterations)

2. $S_{i}=\arg \max \left\|\left(\Phi^{\prime} r_{i}\right)\right\|_{2}^{2} \quad$ (Choose L best indices)

3. $T_{i}=S_{i} \cup S_{\text {stemp }} \quad$ (Construct a temporary index set)

4. $\hat{x}_{i}=\Phi_{T \mathrm{i}}^{\dagger} y$

(Perform estimation)

5. $r_{i}=y-\Phi \hat{x}_{i}$

(Update the residue)

6. $S_{\text {stemp }}=\arg \max \left|\hat{x}_{i}\right|$

(Choose L best indices)

7. $\beta_{i}=1-\frac{\left\|r_{i}\right\|_{2}^{2}}{\|y\|_{2}}$

(Determine the step strategy)

8. if $\|y\|_{2}^{2}<\rho$, Let $\hat{x}=\hat{x}_{i}$

(Quit the iteration)

until halting criterion true 
As shown in table 1 , there are three input parameters for the proposed algorithm. The input parameters include the sensing matrix $\Phi$, the measurement vector $y$ and the initial step size $L_{0}$. And it consists of eight main steps. The most critical step is the seventh step. That can determine which step strategy to be used by judging the parameter $\beta_{i}$. In contrast to other greedy algorithms, the support update $T_{i}$ in the proposed procedure is dynamic in a sense that any index can be added to, or removed from, the estimated support $S_{i}$ at each iteration.

Similar to other greedy algorithms, the JsTMP algorithm also calculate correlation coefficient $\left|\Phi^{\prime} r_{i}\right|$ in the first step. This step is referred to as correlation maximization (CM). We need to make it clear. In each iteration, the $\mathrm{CM}$ operation costs $\mathrm{O}(\mathrm{mn})$ computations and the complexity of the projection is marginal compared with the $\mathrm{CM}$. So the computational complexity of the algorithm proposed is $\mathrm{O}(\mathrm{mn})$. The updated support $S_{i}$ is then calculated as the indices of the $L_{i}$ approximation coefficients with largest magnitude values. These updated supports are then merged with the previously backtracking index set $S_{\text {stemp }}$ to construct the set of indices set $T_{i}$. The sub matrix $\Phi_{T_{i}}$ is used for calculating the least-squares coefficients to estimation the signal in the fourth step in Table 1 . Then the residue $r_{i}$ is updated by calculating $y-\Phi \hat{x}$ in the following iteration. According to the conditions of the fourth step, optimize the step size strategy. Finally, the JsTMP algorithm will terminate when the energy of residue $r_{i}$ satisfies $\left\|r_{i}\right\|<10^{-6}$.

\section{IV.EXPERIMENTAL CLASSIFICATION RESULTS AND ANALYSIS}

In this section, some experiments are conducted to illustrate the performance of the proposed JsTMP algorithm. The proposed algorithm is compared with other greedy algorithms which are include OMP, ROMP, SP, StOMP and CoSaMP. Experimental platform was MATLAB R2013b. A Gaussian random sparse signal with length of $\mathrm{N}=256$ is used to test the probability of exact reconstructionof the proposed JsTMP algorithm. Each sparsity K runs 1000 simulations to get the probability of exact reconstruction.

Fig.4 shows each algorithm's recovery probability under different sparsity level. It can be seen from the simulation results that compared with other algorithms, the proposed algorithm can reconstruct the sparse signal with higher precision for different sparsity level. When sparsity level $\mathrm{K}>60$, all the algorithms start to fail besides the proposed JsTMP. Even if the sparsity level $\mathrm{K}=60$, the probability of reconstruction of JsTMP algorithm is almost $70 \%$. So this also illustrates the superiority of adopting the joint step strategy. And it can be found that when $\mathrm{K}=45$, the probability of exact reconstruction of the proposed algorithm is still close to $100 \%$, but other algorithms begin to decay.

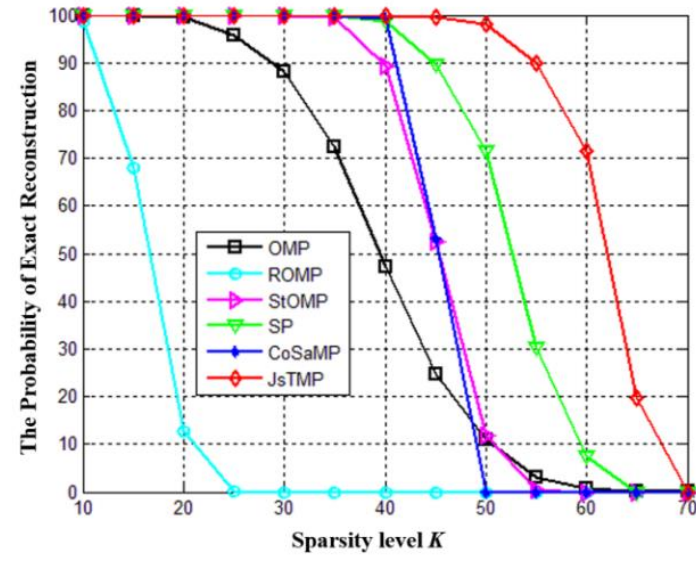

Fig.4 The probability of exact reconstruction with $K=[10,15,20$, $25, \ldots ., 70]$ (test signal is $N=256$ in length, the number of measurements is $M=128$, and the Initial fixed step size $L_{0}=5$ (the red line in the figure)

The error of the algorithm is defined as the Mean Squared Error (MSE), which is shown in (10), where $\hat{x}$ is the reconstructed signal and $x$ is the original signal. $\|.\|_{2}^{2}$ is the energy of signal. We choose the sparsity level $K$ from 10 to 60.1000 simulations were independently carried out for calculating the MSE of the reconstructed signal $\hat{x}$ for different algorithms. Fig. 5 presents the MSE of the reconstructed signal.

$$
M S E=\frac{\|x-\hat{x}\|_{2}^{2}}{\|x\|_{2}^{2}}
$$

As the following Fig. 5 shows that the proposed algorithm is better than other greedy algorithms (OMP, SP, StOMP and so on) in the MSE of the reconstructed signal. So the JsTMP algorithm can accurately reconstruct the original signal.

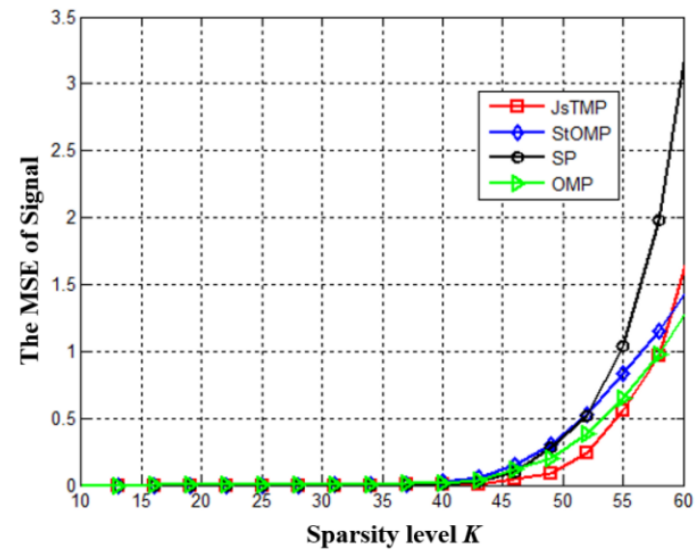

Fig.5 The MSE of exact reconstruction with $K=[10,15,20,25$, $\ldots, 60]$ (test signal is the Gaussian random sparse signal of $N=256$ in length, the number of measurements is $M=128$ (the red line in the figure)).

We depict the number of measurements $(M)$ required by the JsTMP algorithm for recovering Gaussian random sparse signal with length $N=256$. The sparsity levels are chosen from $K=4$ to $K=36$. And the probability of exact recovery signal is averaged over the 1,000 trials, It can be seen from the Fig. 6 that the proposed algorithm can 
accurately reconstruct the signals with different sparsity level $K$.

It can be seen from the Fig. 7 that the probability of exact reconstruction of JsTMP algorithm is better than that of OMP, SP, CoSaMP and StOMP for Gaussian random sparse signal. The probability of exact reconstruction of JsTMP algorithm when measurement varies is higher than that of other algorithms and JsTMP algorithm which does not require the prior knowledge of sparsity $K$. It is concluded that the proposed algorithm has obvious advantages over the rest greedy algorithms when the signal sparsity changes. When the measurement is 70 , the exact reconstruction rate of the proposed algorithm is at least $10 \%$ higher than that of other algorithms.

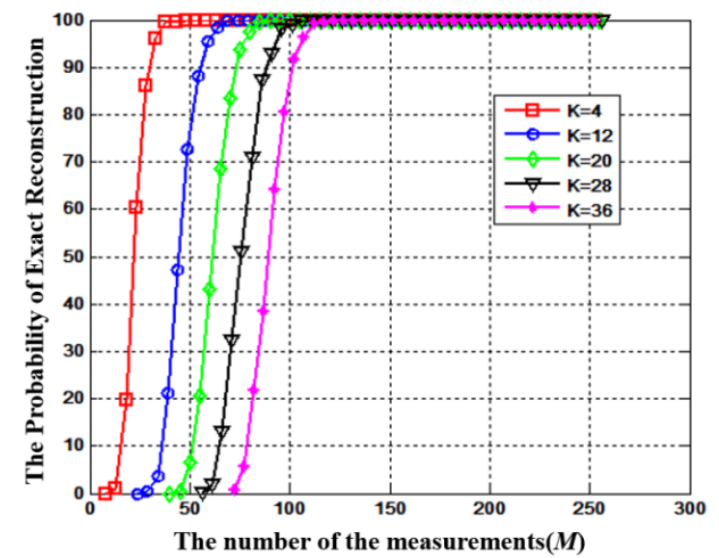

Fig.6 Probability of exact recovery signal with proposed algorithm. the original signal has length of $N=256$ with sparsity level $K=4,12,20,28,36$ non-zero entries.

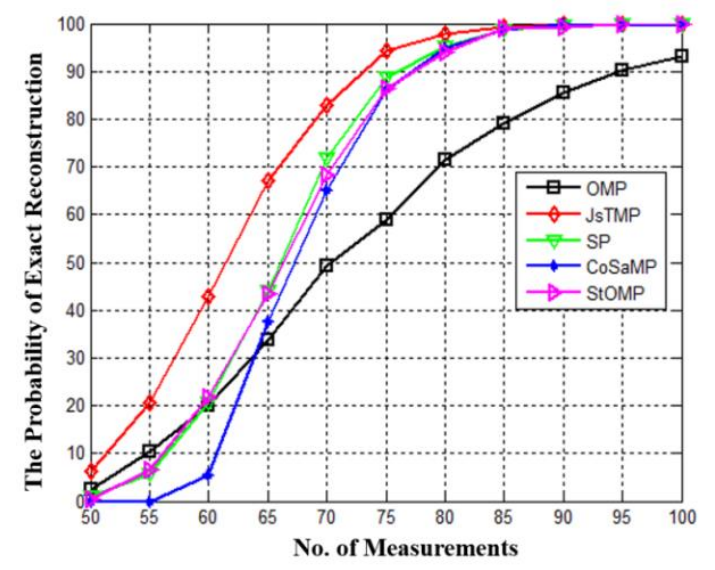

Fig.7 The probability of exact reconstruction with the measurements $M=[50,55,60, \ldots ., 100]$ (test signal is the Gaussian random sparse signal of $N=256$ in length, the sparsity level $K=20$ )

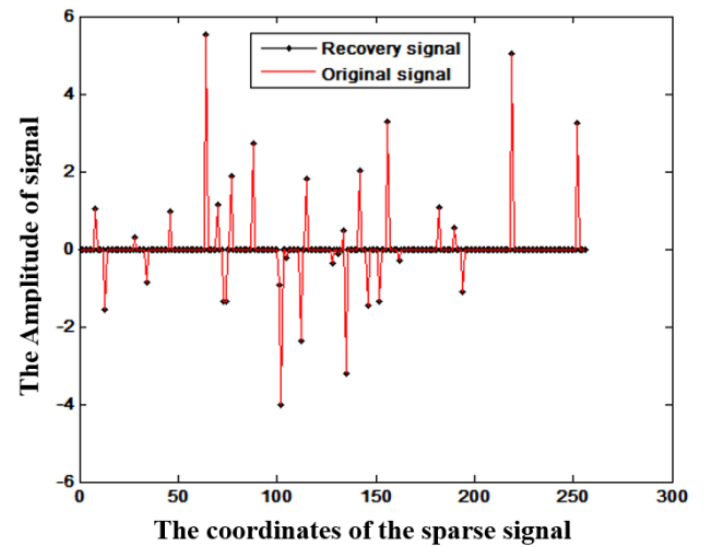

(a)

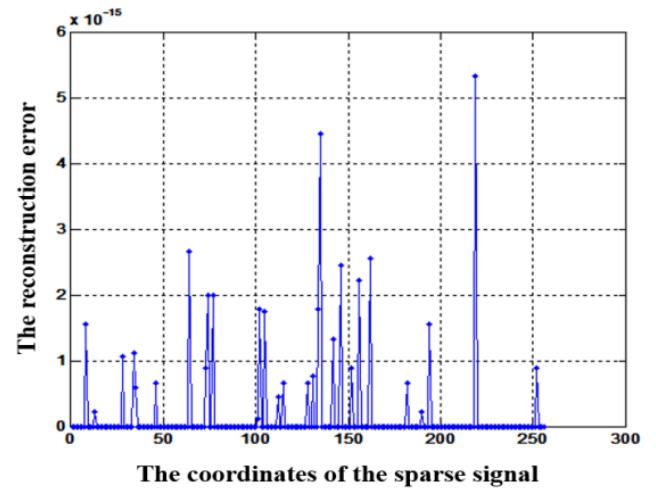

(b)

Fig.8 The reconstruction of the sparse signal in time domain(a), the reconstruction error (b) (test signal is the Gaussian random sparse signal of $N=256$ in length, the sparsity level $K=30$ )

In Fig.8, we show the effect of the reconstructed signal in the time domain. And the reconstruction error is set to $|x-\hat{x}|$ in time domain. (a) is a comparison between the original signal and the reconstructed signal in the time domain. It can be seen from (b) that the value of vertical axis in the reconstructed error is on the order of magnitude of 10-15. In practice, such a small reconstruction error is negligible. That is to say that the proposed algorithm can accurately reconstruct the original signal.

\section{CONCLUSION}

In this paper, a novel joint step-size matching pursuit algorithm(JsTMP) is proposed. The JsTMP algorithm uses the fixed step size and dynamic step size to adjust the number of selected indexs, while using backtracking strategy to remove the wrong index. Compared with the other existing algorithms, the proposed JsTMP algorithm can quickly converge to the real sparsity of the target signal and accurately reconstruct the original signal. Simultaneously, it does not require the prior knowledge of the sparsity level $\mathrm{K}$. The multiple simulation results demonstrate that JsTMP can precisely reconstruct the original signal with high probability in this paper. For those advantages, the proposed algorithm has broad application prospects and a higher guiding significance for the research on sparse signal reconstruction. 
Author Contributions: Dongxue propose the methodology; Zengke wirtie software and validate the proposed method; Dongxue writes this paper; Zengke reviews and edits it.

Funding: The research is supported by the National Nature Science Foundation of China (No. 61771262).

Conflicts of interest: The authors declare no conflict of interest.

\section{REFERENCES}

[1] D.L.Donoho, “Compressed Sensing,” IEEE Trans. Information Theory, vol. 52, no. 4, pp. 1289-1306, Apr 2006.

[2] E. J. Candes, M. B. Wakin, "An introduction to compressive sampling," IEEE Siganl Process., vol 25, no. 2, pp. 21-30, Mar 2008.

[3] E. Candes, J. Romberg, T. Tao, "Stable Signal Recovery From Incomplete and accurate Measurement," Communication on Pure and Applied Mathematics, vol 59, no. 8, pp. 1207-1223, 2006.

[4] Zhu J, Zhang Q, Meng X, et al. Vector approximate message passing algorithm for compressed sensing with structured matrix perturbation[J]. Signal Processing, 2020, 166: 107248 .

[5] Qiu W, Zhou J, Fu Q. Jointly Using Low-Rank and Sparsity Priors for Sparse Inverse Synthetic Aperture Radar Imaging[J]. IEEE Transactions on Image Processing, 2019, 29: 100-115.

[6] Leong Z, Tan T L. Robust cluster expansion of multicomponent systems using structured sparsity[J]. Physical Review B, 2019, 100(13): 134108.

[7] $\mathrm{Li} \mathrm{Y,} \mathrm{Li} \mathrm{K,} \mathrm{Zhang} \mathrm{C,} \mathrm{et} \mathrm{al.} \mathrm{Learning} \mathrm{to} \mathrm{reconstruct} \mathrm{computed} \mathrm{tomography} \mathrm{(CT)} \mathrm{images}$ directly from sinogram data under a variety of data acquisition conditions[J]. IEEE transactions on medical imaging, 2019.

[8] D. L. Donoho, Y. Tsaing, I. Drori, et al. "Sparse solution of underdetermined systems of linear equations by stagewise orthogonal matching pursuit," IEEE Trans. Information Theory, vol. 58, no. 2, pp. 1094-1121, Feb 2012.

[9] Wei Dai, Olgica Milenkovic. "Subspace Pursuit for Compressive Sensing Signal Reconstruction," IEEE Transactions on Information Theory, vol. 55, no.5, pp. 2230 - 2249, April 2009.

[10] Wen, JM, Wang, J, Zhang, QY. "Nearly Optimal Bounds for Orthogonal Least Squares," IEEE TRANSACTIONS ON SIGNAL PROCESSING, vol. 65, no.20, pp. 5347-5356, OCT 2017.

[11] Cohen Albert, Dahmen Wolfgang, DeVore Ronald. "Orthogonal Matching Pursuit Under the Restricted Isometry Property," CONSTRUCTIVE APPROXIMATION, vol. 45, no. 1, pp. 113-127, Feb 2017.

[12] Voroninski V, Xu ZQ. "A strong restricted isometry property, with an application to phaseless compressed sensing," Applied and Computational Harmonic Analysis, vol. 40, no. 2, pp. 386-395, Mar 2016.

[13] Chiani-M, Elzanaty-A, Giorgetti-A, "Analysis of the Restricted Isometry Property For Gaussian Random Matrices," in 2015 IEEE Global Communications Conference (GLOBECOM), pp.6,San Diego, CA. Dec 06-10,2015.

[14] Salahdine, Kaabouch, EI Ghazi, "A Bayesian recovery technique with Toeplitz matrix for compressive spectrum sensing in cognitive radio networks", International journal of communication Systems. vol. 30, no. 15, pp.1-9, Feb 2017.

[15] Haupt J, Bajwa W U, Raz G, et al. Toeplitz compressed sensing matrices with applications to sparse channel estimation[J]. IEEE transactions on information theory, 2010, 56(11): 5862-5875.

[16] Barata J C A, Hussein M S. The Moore-Penrose pseudoinverse: A tutorial review of the theory[J]. Brazilian Journal of Physics, 2012, 42(1-2): 146-165.
Creative Commons Attribution License 4.0 (Attribution 4.0 International, CC BY 4.0)

This article is published under the terms of the Creative Commons Attribution License 4.0

https://creativecommons.org/licenses/by/4.0/deed.en US 